\title{
EDITORIAL
}

\section{Thrombolysis: too old and too young}

\section{S G Ball}

\section{Elderly patients are different from their younger counterparts when it comes to thrombolysis}

T hrombolysis is established therapy for acute myocardial infarction. The recent National service framework for coronary heart disease ${ }^{1}$ seeks proof of its rapid delivery in all hospitals in England in the near future. Witnessing a stroke, of which the majority are fatal, after delivering a thrombolytic agent brings home to clinicians how narrow the therapeutic benefit from these agents can be. Faced with the individual patient, the prospect of minor gain against the rare potential for death or serious disability weighs heavily in decision making; doctors treat patients not populations. An article by Thiemann and colleagues ${ }^{2}$ suggested that elderly patients have nothing to gain from thrombolysis, indeed they may be harmed (fig 1). The claims have raised an interesting debate. Can such non-randomised studies, however large the patient numbers and elegant the statistics, replace information from the randomised trial? Probably not, yet extrapolation from trials that include low risk, relatively young patients may be equally inappropriate.

Age appears not to affect in any fundamental way the pathology of the culprit lesion of acute myocardial infarction. However, the extent of associated disease in the coronaries is likely to be greater and co-morbid conditions are common. The dramatic rise of mortality with age makes clear that elderly patients are different in a major way from their younger counterparts. Similarly the higher mortality of those after myocardial infarction, when not entered in to trials of thrombolysis, even when matched for age, indicate substantial differences between "real life" and trial findings.

What then is the evidence from the randomised trials for using thrombolytics in the elderly? This important clinical question is addressed in the article by Estess and Topol in this issue of Heart. ${ }^{3}$ Immediately apparent is that information in the elderly is sparse and poorly reported, making it difficult for clinicians to make "evidence based" decisions. This is surprising given that 30\% of infarcts occur in those over the age of 75 years. However, since $70 \%$ of these elderly patients will not have ST segment elevation associated with their infarction, the actual number even to be considered for thrombolysis falls dramatically. Published evidence comparing thrombolysis against placebo or control treatment is available from the fibrinolytic therapy trialists (FTT) overview. ${ }^{4}$ However, of the 58600 patients in nine trials, only 5788 were over the age of 75 years. At 30 days there was no evidence of a significant benefit from thrombolysis (10 lives saved per 1000 treated but with a $95 \%$ confidence interval
(CI) from 16 lost to 36 saved). In contrast younger patients had a greater and statistically significant relative risk reduction, but with a much lower absolute mortality leading overall to a saving of about 18 lives per 1000 treated ( $95 \%$ CI 12 to 24 ). Nevertheless, given the high absolute mortality of those over 75 years, even with the smaller risk reduction, the wide confidence interval permits a substantial potential overall benefit. A similar but again non-significant long term benefit to older patients, split above and below age 70 years, is shown in a subgroup of the second international study of infarct survival (ISIS-2) trial (4l saved per 1000 but with $95 \%$ CI of 7 lost to 89 saved in those over 70 years, compared with benefit to those under 70 years of 23 lives saved for 1000 treated with $95 \%$ CI 4 to 42 ).

Current practice dictates thrombolysis for those "within 12 hours and with ST elevation or new bundle branch block". Do we have evidence for treatment of this group in the elderly population? Unpublished data from the FTT secretariat suggest no significant heterogeneity of the proportional reduction in 35 day mortality according to age. In a cohort of 3332 over age 75 years with ST elevation or new bundle branch block, mortality was reduced from $29.4 \%$ to $26.0 \%$, a significant $(p=0.03) 15 \%$ relative risk reduction. Although lower than the proportional reduction in risk seen in the younger cohorts because of the much higher risk of this age group, the absolute saving of lives is substantial ( 34 lives per 1000 treated) but with wide $95 \%$ CIs (from 2 to 66 lives saved). The corollary is that 2456 elderly patients (5788 minus 3332) thrombolysed outside the "within 12 hours, ST elevation or new bundle branch block" indications, reported in the FTT review were harmed substantially. No information is available yet on the steepness of the benefit against the time delay for the elderly.

". . . in the elderly only those with early presentation and clear cut new ST elevation, especially if in the anterior leads or new bundle branch block, should be thrombolysed"

What is the thinking clinician to do? Often the difficult decision on thrombolysis is left to the least experienced member of the team. Such individuals are under pressure to achieve better times to thrombolysis. Since thrombolysis is often given in the emergency room, the decision makers may

Abbreviations: FTT, fibrinolytic therapy trialists; ISIS-2, second international study of infarct survival; t-PA, tissue plasminogen activator 


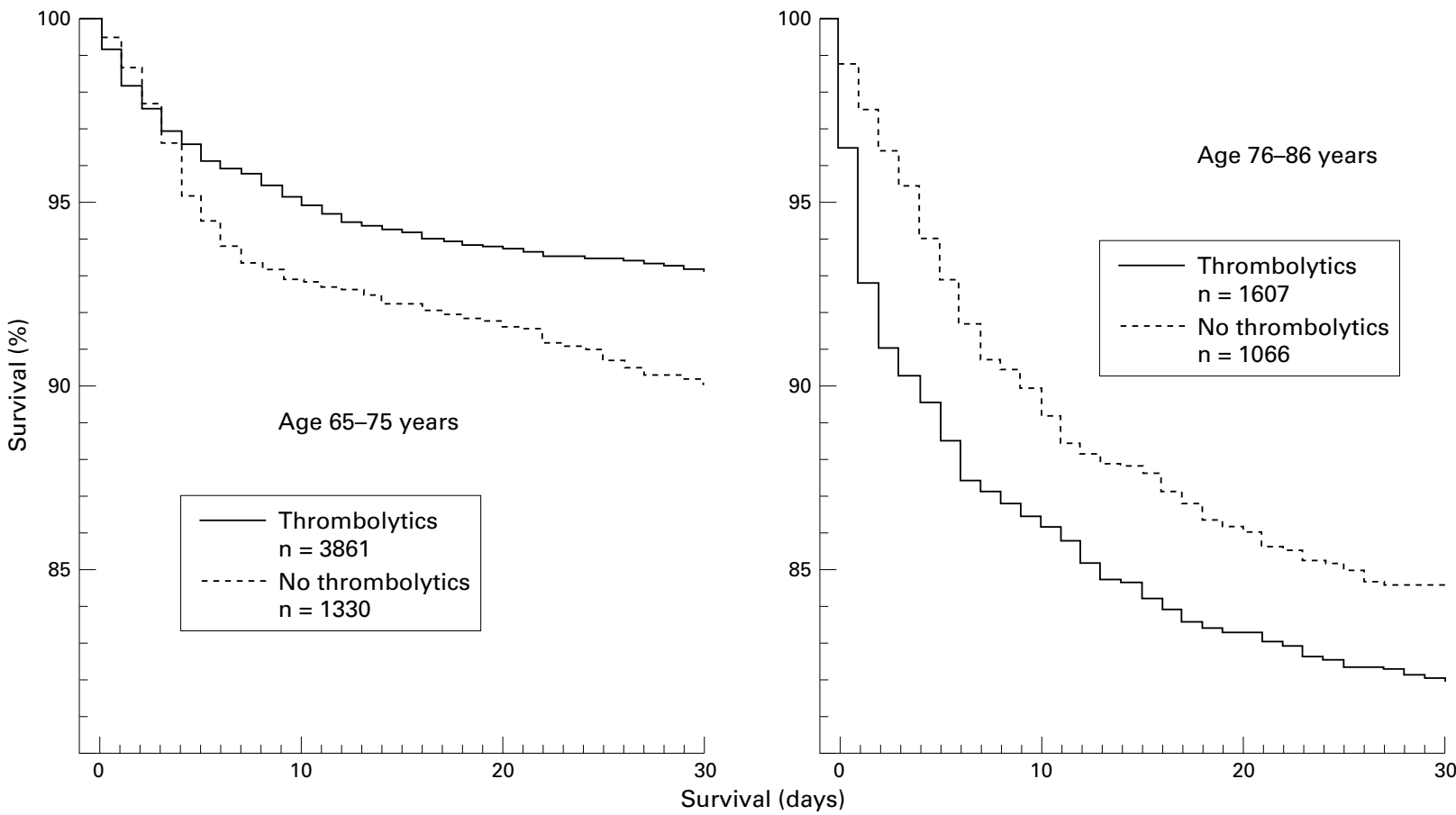

Figure 1 Observed mortalities according to age in patients thought likely to benefit from fibrinolysis - that is, within six hours and with anterior ST elevation. Mortality in the younger age group was $6.8 \%$ in those thrombolysed against $9.8 \%$ in those not. However, in patients aged $75-85$ years the reverse was observed, with a higher $18 \%$ mortality in those thrombolysed compared with $15.4 \%$ in those not. Adapted from Thiemann et al, Circulation 2000;101:2239-46, with permission.

never witness the consequence of their decisions. For the moment the clear message should be that particularly in the elderly only those with early presentation and clear cut new ST elevation, especially if in the anterior leads or new bundle branch block, should be thrombolysed. How early is early might be asked? Extrapolation to elderly patients from the overall results indicates that within four hours makes thrombolysis a serious consideration, whereas beyond six leaves doubt. The risk of stroke, unlike the saving of myocardium, is not time dependent but does relate clearly to age with tissue plasminogen activator ( $\mathrm{t}-\mathrm{PA}$ ) though less obviously so (if at all) with streptokinase. Clinical severity and certainty of timing of the precipitating event, taking account perhaps more of the "biological age and co morbid conditions" than the chronological age, must influence any treatment decision. Such suggestions do not lend themselves well to protocols implemented by the less experienced; equally advocacy of such caution offers no excuse for failing to implement treatment rapidly where evidence clearly indicates benefit. When doubt exists then experienced advice should be sought. Importantly in this context the article by Estess and Topol highlights areas for concern that can be dealt with by protocol. The weight of patients may be a critical issue when t-PA is used, not merely for its own administration but particularly for the dose of heparin for co-administration. High blood pressure levels are also related to the subsequent complication of stroke. These are areas in which clinicians could be more vigilant. Elderly patients with a large anterior myocardial infarction of recent onset leading to concomitant low blood pressure have potentially much to gain from successful thrombolysis administered optimally. Nevertheless, high rates of thrombolysis administered rapidly without regard to the characteristics of the population under treatment should not be regarded as a measure of high quality care.

\section{REFERENCES}

1 Department of Health. National service framework for coronary heart disease: modern standards and service models. London: Department of Health, 2000

2 Thiemann DR, Coresh J, Schulman SP, et al. Lack of benefit for intravenous thrombolysis in patients with myocardial infarction who are older than 75 years. Circulation 2000;101:2239-46.

3 Estess JM, Topol EJ. Heart 2002;87:IN THIS ISSUE

4 Fibrinolytic Therapy Trialists (FTT) Collaborative Group. Indications for fibrinolytic therapy in suspected acute myocardial infardction: collaborative overview of early mortality and major morbity result from all randomized trials of more than 1000 patinets. Lancet 1994;343:311- 